\title{
Primary care physicians' understanding and utilization of pediatric exome sequencing results
}

\author{
Sarah E. Mazzola ${ }^{1,2}$ | Bridget O'Connor ${ }^{3}$ | Beverly M. Yashar ${ }^{2}$
}

${ }^{1}$ Genomic Medicine Institute, Cleveland Clinic Foundation, Cleveland, Ohio

${ }^{2}$ Department of Human Genetics, University of Michigan, Ann Arbor, Michigan

${ }^{3}$ Division of Pediatric Genetics, University of Michigan, Ann Arbor, Michigan

\section{Correspondence}

Sarah E. Mazzola, Genomic Medicine Institute, Cleveland Clinic Foundation, 9500 Euclid Ave, R4, Cleveland, OH 44195.

Email:mazzols@ccf.org

\section{Funding information}

National Society of Genetic Counselors Research Special Interest Group; Michigan Association of Genetic Counselors, Inc

\begin{abstract}
Optimizing exome sequencing (ES) utility requires effective communication and collaboration between primary care physicians (PCPs) and genetics healthcare providers (GHP). To explore how PCPs use ES results to coordinate multipart management plans for complex pediatric patients, we assessed result understanding and utilization. Twenty-seven PCPs of pediatric patients with ES results from a genetics clinic completed a mixed methods 45-question survey measuring perceived genetics knowledge, confidence performing genetics tasks, understanding of ES technology and results, and expectations of GHP. Quantitative and qualitative data analysis classified by ES result types generated descriptive statistics, Pearson correlation coefficients, and common themes. Forty-five-percent of PCPs interpreted variant of uncertain significance results as diagnostic (implementing management changes and recommending familial testing). Most PCPs (85\%) identified positive ES results impacts, but only $65 \%$ indicated ES was beneficial to care. The majority (74\%) expected GHP and patients' families to assume follow-up care responsibility and future ES results re-interpretations. Limited knowledge may be a factor, as $59 \%$ desired more patient care information from GHP. Our results suggest optimizing continuity of care and collaboration for pediatric patients with ES results requires additional communication between GHP and PCPs, along with continuing genetics education for PCPs aimed at improving genetic literacy.
\end{abstract}

\section{KEYWORDS}

exome sequencing, genetics, genetics services, pediatric genetics, pediatrics, primary care, provider communication

\section{1 | INTRODUCTION}

Pediatric patients embarking on genetic diagnostic odysseys often have complex medical needs and their primary care physicians (PCPs) play critical care roles by serving as the medical home that coordinates their long-term multipart management plans (Genetics in Primary Care, 2018; Lantin-Hermoso et al., 2017). As new genetic testing technology is developed and increasingly implemented, PCPs' responsibilities for these patients and their families will continue to grow and proper patient care will depend on appropriate levels of genetic literacy and collaboration with genetics healthcare providers (GHP), clinical specialists who evaluate, diagnose, and provide information and recommendations regarding genetic conditions.

The introduction of exome sequencing (ES) as a diagnostic test has provided diagnoses to many pediatric patients who had exhausted all other genetic test options (Biesecker \& Biesecker, 2014; Iglesias et al., 2014; Lazaridis et al., 2016; Lee et al., 2014; Sawyer et al., 2016). With a diagnostic rate ranging from $20 \%-$ $34 \%$, compared to that of chromosomal microarray (CMA) at $12 \%-$ $22 \%$, the clinical usefulness and diagnostic value of ES in certain 
pediatric populations is clear (Battaglia et al., 2013; Biesecker \& Biesecker, 2014; Coulter et al., 2011; Henderson et al., 2014; Iglesias et al., 2014; Lazaridis et al., 2016; Lee et al., 2014; Miller et al., 2010; Sawyer et al., 2016). Although useful, this complex genetic test has resulted in novel challenges, ranging from variant interpretation to determining management for novel conditions, for GHP who order the test and PCPs who receive their patients' results (Biesecker \& Biesecker, 2014; Iglesias et al., 2014; Lee et al., 2014; Nguyen \& Charlebois, 2015; Tetreault, Bareke, Nadaf, Alirezaie, \& Majewski, 2015).

In order to utilize the various complicated possible genetic testing results, PCPs need to maintain a sufficient understanding of genetics to ensure proper implementation and communication of recommendations regarding test results from GHP into their patient's everyday care. PCPs have reported limited general genetics knowledge and skills along with shortcomings related to confidence, comfort, and ability to perform specific genetics related tasks (Mikat-Stevens, Larson, \& Tarini, 2015). Furthermore, while a subset of PCPs indicated that genetics evaluations were useful for their patients, only approximately half of these PCPs felt competent to provide healthcare for patients related to genetics following their genetics evaluations (Rinke et al., 2013). This perspective was mirrored by patient reports ranking their PCPs' overall genetics knowledge as poor (Harvey et al., 2007). As pediatric genetics patients often present with multiple physical and cognitive differences, PCPs low reported genetics knowledge and lack of confidence in their abilities to provide care that is informed by genetics results can be detrimental to the continuous management and care for these patients.

Continuity of care is essential for pediatric genetics patients as they maintain care with their medical home, the PCPs, following a limited number of genetics appointments. The American Academy of Pediatrics Council on Children with Disabilities acknowledges several barriers to effective care coordination of PCPs as the medical home: lack of knowledge and information, lack of communication among healthcare professionals, and lack of clearly defined roles for each member of the practice team (American Academy of Pediatrics Council on Children with Disabilities, 2005). Consequently, in order to improve patient care, it is critical to identify ways to promote communication and collaboration between PCPs and GHP. This study assessed PCPs' understanding and utilization of ES results in the management of their pediatric patients and families with an objective of improving continuity of care for pediatric genetics patients by identifying areas to improve communication and collaboration between PCPs and GHP.

\section{2 | METHODS}

\section{1 | Participants}

We recruited PCPs whose patient received an ES test result ordered by the University of Michigan Health Services- Division of Pediatric Genetics between February 2013 and October 2016
( $n=308)$. To increase PCP's survey responses' specificity, the following patients/PCPs were excluded: three or more variants detected by ES ( $n=40)$, GHP reinterpreted the laboratory reported result $(n=2)$, deceased ( $n=2$ ), and more than one patient with an ES result for a single PCP $(n=50)$. We prioritized inclusion of patients from negative and pathogenic variant result groups to attempt recruitment of an equal number of PCPs for each ES result type. A total of 214 PCPs were mailed a recruitment letter that included the ES result with the laboratory report interpretation on their named patient, and a link to an online survey. This study was approved by the University of Michigan Institutional Review Board (HUM00118547).

\section{2 | Instrumentation}

To assess PCPs' knowledge about and use of ES results in their patients' care, we developed a mixed methods study instrument including modified questions from validated instruments that evaluated PCPs' perspectives and understanding of genetics, ES in general, and applying genetic test results to patient's care (Data S1; Jez, Martin, South, Vanzo, \& Rothwell, 2015; Pearl et al., 2011; Reiff et al., 2015; Rinke et al., 2013; Saam, Gudgeon, Aston, \& Brothman, 2008). The 45-question instrument focused on five domains: physician demographics (6 questions), self-assessed genetics knowledge and confidence (14 questions), understanding of ES results (6 questions), patient and family healthcare management (6 questions), communication with patients and their families (3 questions), and collaboration with GHP (10 questions). Response options included 5-point Likert scales that assessed PCP's genetics knowledge (1: none/minimal, 2: basic, 3: proficient, 4: advanced, 5: superior) and confidence performing genetics related tasks (discussing or making genetics referrals, discussing or ordering genetic testing, interpreting or communicating genetic testing results; 1 : very uncomfortable to 5: very comfortable), as well as true/false, select all that apply, and free response questions to assess participants' understanding of ES results, communication with patients and families, and collaboration with GHP. The first question asked the PCP to identify their patient's result (pathogenic variant, variant of uncertain significance (VUS), negative) and to disregard any incidental findings. Participants provided informed consent prior to completing the survey. Prior to finalization, the instrument was piloted with a pediatric geneticist, a pediatric genetics resident, and an otolaryngologist who had received an ES patient result.

\section{3 | Data analysis}

Quantitative analysis using descriptive statistics, ANOVA, and Pearson correlation coefficients was completed using R software with the help of statistical consultants at the University of Michigan Center for Statistical Consultation and Research. Likert scale questions for genetics knowledge and confidence were analyzed individually and summed to generate separate knowledge and confidence scores for each PCP. Qualitative analysis of open text responses 
were completed by three team members utilizing structural and descriptive coding methods to identify common themes (Saldaña, 2013). An error in survey administration led to partial survey completion by one respondent. This respondent's results are included in the data analysis for the completed portions and results for relevant sections reflect this variation in participant numbers. ES results were defined as either "laboratory reported result" for the laboratory issued report or "PCP reported result" for the PCP's survey response. Analyses based on result type utilized PCP reported results.

\section{3 | RESULTS}

\section{1 | PCP demographics}

Of the 214 recruited PCPs (laboratory reported results: 51 with pathogenic variant, 18 with pathogenic variant and VUS, 94 with VUS, 51 with negative results), 27 PCPs responded (laboratory reported results: 9 with pathogenic variant, 14 with VUS, 4 with negative results). Each participating PCP provided responses based on a single patient result. The overall response rate was $12.6 \%$. Among the 27 responding PCPs, the majority (63\%) of PCPs have been in practice for more than 10 years and for $9 / 27$ (33\%) their last genetics training was $10+$ years ago (Figure 1). Additionally, $7 / 27$ (26\%) PCPs' last genetics training was in medical school, and from those, $6 / 7$ (86\%) of these PCPs' medical school training was $10+$ years ago.

To assess respondents' genetics understanding, PCPs self-rated their knowledge of eight genetics subjects and comfort performing genetics related tasks. The mean totaled genetics knowledge scores for respondents was 23.2 (range $=16-38$; Table 1). All respondents indicated that they were somewhat comfortable or very comfortable discussing and making referrals to GHP (mean $=8.9$, range $=8-10)$. The mean self-assessed comfort score performing genetic testing-related tasks was 12.9 (range $=8-19$ ). We found participants with higher knowledge scores were more likely to have higher comfort scores for performing genetics referral tasks $(r=.54, p=.003)$ and genetic testing related tasks $(r=.69$, $p=<.001)$. We found that those practicing as PCPs for shorter time periods were more likely to have higher knowledge scores $(r=-.41, p=.034)$ and higher comfort performing genetic testing tasks scores ( $r=-.25, p=.218$ ). PCPs who attended a more recent genetics training were also more likely to have higher knowledge scores ( $r=-.42, p=.029)$ and higher comfort performing genetic testing tasks scores $(r=-.59, p=.001)$. One-way ANOVA analysis did not identify any statistically significant differences between ES result type and knowledge scores $(F(2,24)=2.918, p=.073)$, comfort performing referrals to GHP scores $(F(2,24)=0.691$, $p=.511$ ), or comfort performing genetic testing-related tasks scores $(F(2,24)=1.503, p=.243)$.

\subsection{Understanding of ES test results}

To assess PCPs' understanding of their patient's genetic results, they were asked to state what type of ES result their patient received. Of the 27 respondents (PCP reported results: 10 with pathogenic variant, 12 with VUS, 5 with negative results), most PCPs (93\%) correctly identified their patient's ES result type. However 2/14 PCPs (14\%) incorrectly reported their patients' VUS results: one PCP indicated that their patient received a pathogenic result, and the other a negative result. For these two study participants, subsequent responses were evaluated according to ES result type they indicated for their patient.

PCPs shared their perspectives on the impact of ES on their patient's care (Table 2). The majority of PCPs overall $(17 / 26,65 \%)$ and of each result type group (pathogenic $8 / 10,80 \%$; VUS 6/11, 55\%; negative $3 / 5,60 \%$ ) agreed with the statement that ES was beneficial to their patient's care. To deepen our understanding of the PCPs' views of ES, all PCPs were asked to identify the most and least beneficial outcomes of receiving ES test results. Of the 22 PCPs who provided reasons, 24 benefits were identified overall and condensed into four themes: (a) family emotional support (12/24, 50\%), (b) identifying a genetic diagnosis $(6 / 24,25 \%)$, (c) ruling out a genetic diagnosis $(3 / 24,13 \%)$, and (d) providing medical management $(3 / 24,13 \%)$. Of the 23 PCPs who identified unfavorable outcomes, 27 outcomes were provided and condensed into four themes: (a) inability to alter
Participant Demographics

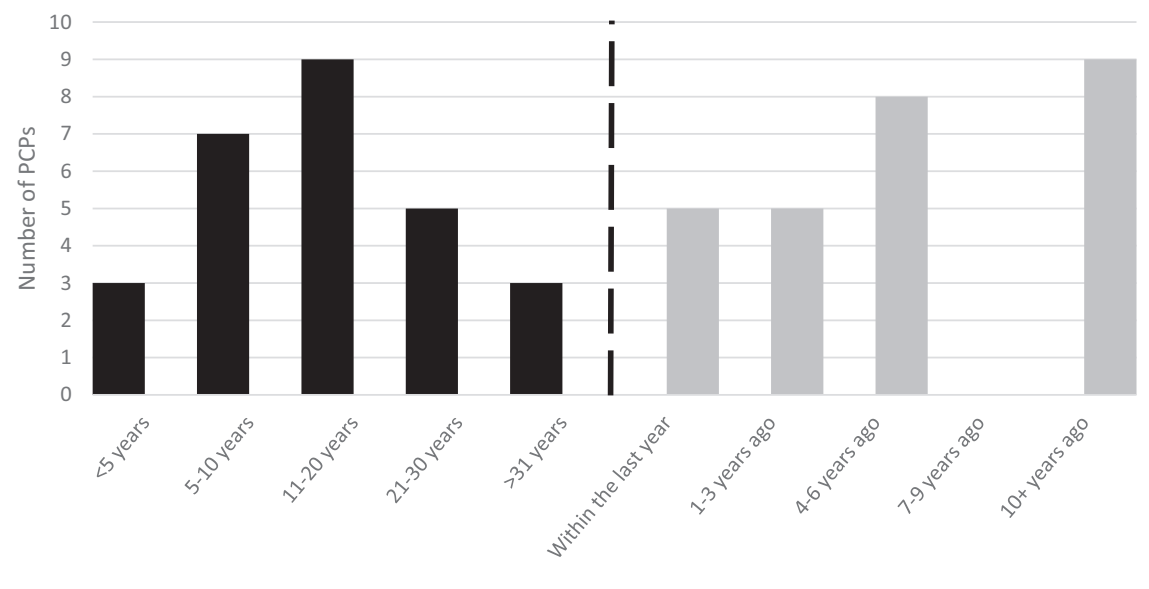

- Time Practicing as a PCP $\square$ Most Recent Genetics Training
FIGURE 1 Legend: Demographic information of Primary Care Physicians (PCPs) divided by years of experience practicing as a PCP and by the timing of $P C P$ 's last genetics training 
TAB LE 1 Primary care physicians genetics knowledge and confidence performing genetics tasks

\begin{tabular}{|c|c|c|c|c|}
\hline Assessment domain & \multicolumn{4}{|c|}{ Type of exome sequencing result received } \\
\hline Genetics knowledge score (Possible range 8-40) & $25.3(16-33)$ & $23.6(16-38)$ & $17.8(16-19)$ & $23.2(16-38)$ \\
\hline $\begin{array}{l}\text { Confidence performing genetics referral tasks }{ }^{a} \\
\text { (Possible range } 2-10 \text { ) }\end{array}$ & $9.1(8-10)$ & $8.8(8-10)$ & $8.6(8-10)$ & $8.9(8-10)$ \\
\hline $\begin{array}{l}\text { Confidence with genetic testing tasks }{ }^{b} \text { (Possible } \\
\text { range 4-20) }\end{array}$ & $14.1(8-19)$ & $12.8(9-18)$ & $11.0(8-16)$ & $12.9(8-19)$ \\
\hline
\end{tabular}

Note: Means are displayed with the reported range in parentheses following. " $n$ " in each column equals the number of PCPs with the PCP reported result type.

${ }^{a}$ Genetics referral tasks include discussing or making referrals to genetics.

${ }^{b}$ Genetic testing tasks include discussing or ordering genetic testing and interpreting or communication genetic test results.

management (11/27, 41\%), (b) not receiving a genetic diagnosis ( $7 / 27$, $26 \%)$, (c) receiving uncertain results $(5 / 27,19 \%)$, and (d) perceived financial burden (4/27, 15\%). Although most PCPs could identify both benefits and limitations of ES for their patient and their family, 3/11 (27\%) PCPs with VUS results were unable to identify why ES was beneficial for their patient while 2/10 (20\%) PCPs with pathogenic results were unable to identify any negative connotations of ES for their patient.

To understand how the study participants looked at the ES test results within the context of their patient's diagnostic odyssey, respondents indicated their views on how ES results impacted their patients' ongoing care (Table 2). PCPs with pathogenic results $(9 / 10,90 \%)$ were more likely to agree with the statement that the ES result explains some or all of their patient's symptoms, than PCPs with VUS results (5/11, 45.5\%). The remaining PCPs with VUS results agreed instead with the statement that ES results may explain their patient's symptoms $(5 / 11,45.5 \%)$ or the ES result does not explain their patient's symptoms (1/11, 9\%). Additionally, PCPs with pathogenic results were more likely to agree with the statements that there is a genetic cause for their patient's symptoms and the ES results will be reanalyzed in the future, than PCPs with VUS or negative results, while the minority of PCPs in each result group indicated that there will be more genetic testing in the future for their patient (Table 2).

\subsection{Utilization of ES results in patient/ family healthcare}

To determine how PCPs used ES results in the medical management of their patients, PCPs described what, if any, changes were made. Ten of twenty-six (38\%) PCPs changed management for their patient after receiving the ES result (Table 2). PCPs made a total of 24 management changes for 10 patients. Nine management changes $(9 / 24,38 \%)$ related to patient medical care: medical screenings $(4 / 9,44 \%)$, referral to a specialist $(3 / 9,33 \%)$, new medication regimes $(2 / 9,22 \%)$, while no one discontinued previously recommended screening. Fifteen management changes $(15 / 24,63 \%)$ related to family support: family testing for reproductive reasons (4/15, 27\%), family testing for medical reasons (4/15, $27 \%)$, recurrence risk for the family $(4 / 15,27 \%)$, and improved
TABLE 2 Primary care physicians (PCPs) perspectives on utility of exome sequencing results in patient care

\begin{tabular}{|c|c|c|c|c|}
\hline \multirow[b]{2}{*}{ Assessment statements } & \multicolumn{4}{|c|}{ Type of exome sequencing (ES) result received } \\
\hline & $\begin{array}{l}\text { Pathogenic } \\
(n=10)\end{array}$ & $\begin{array}{l}\text { VUS } \\
\left(n=11^{a}\right)\end{array}$ & $\begin{array}{l}\text { Negative } \\
(n=5)\end{array}$ & All results \\
\hline ES result explains some or all symptoms & $9 / 10(90 \%)$ & $5 / 11(45 \%)$ & - & $14 / 21(67 \%)^{b}$ \\
\hline $\begin{array}{l}\text { There is a genetic cause for my pa- } \\
\text { tient's symptoms }\end{array}$ & 9/10 (90\%) & $7 / 11(64 \%)$ & $1 / 5(20 \%)$ & $17 / 26(65 \%)$ \\
\hline $\begin{array}{l}\text { PCPs encouraging discussion of results } \\
\text { with family members }\end{array}$ & $8 / 10(80 \%)$ & $8 / 11(73 \%)$ & $2 / 5(40 \%)$ & $18 / 26(69 \%)$ \\
\hline ES was beneficial to care & $8 / 10(80 \%)$ & $6 / 11(55 \%)$ & $3 / 5(60 \%)$ & $17 / 26(65 \%)$ \\
\hline Management changes made & $7 / 10(70 \%)$ & $3 / 11(27 \%)$ & $0 / 5(0 \%)$ & $10 / 26(38 \%)$ \\
\hline $\begin{array}{l}\text { ES results will be reanalyzed in the } \\
\text { future }\end{array}$ & $6 / 10(60 \%)$ & $4 / 11(36 \%)$ & $1 / 5(20 \%)$ & $11 / 26(42 \%)$ \\
\hline More genetic testing in the future & $2 / 10(20 \%)$ & $5 / 11(45 \%)$ & $2 / 5(40 \%)$ & $9 / 26(35 \%)$ \\
\hline
\end{tabular}

Note: Data presented in this table (Percentages) indicate the number of participants in each ES result category that agreed with the assessment statement. " $n$ " in each column equals the number of PCPs with the PCP reported result type.

${ }^{a}$ Only 11 of the 12 PCPs with VUS results responded to this set of questions.

${ }^{b}$ Only PCPs with pathogenic and VUS results responded to this question. 
access to services for the family $(3 / 15,20 \%)$. From the management changes made, 3 PCPs with VUS results made $46 \%(11 / 24)$ of the management changes. Management changes made by the PCPs based on ES results was not correlated with the self-reported genetics knowledge scores or comfort performing genetics related tasks scores. We were not able to identify a correlation between patients' clinical presentations and management changes made by PCPs due to medical complexity.

To further classify ES result management implications, PCPs described their interactions with the patients' families. The majority of all PCPs $(18 / 26,69 \%)$ and of pathogenic (8/10, 80\%) and VUS $(8 / 11,73 \%)$ subgroups indicated that they encouraged or will encourage their patient's parents to discuss their child's genetic testing results with other family members (Table 2 ). Among these 18 providers, the rationale for encouraging family conversations included: important information for the family to know (13/18, $72 \%)$, providing/receiving support (11/18, 61\%), acceptance and understanding of the child (10/18, 55\%), help processing the information provided by healthcare professionals $(8 / 18,44 \%)$, and advice (4/18, 22\%)

\section{4 | Provider expectations and needs}

To assess PCP experiences with GHP, PCPs described their communication with GHP and views of follow-up responsibilities (Table 3). Only 11/27 (41\%) PCPs reported receiving sufficient information from GHP to properly manage and counsel their patients and families (Table 3). Among the 59\% (16/27) who felt they did not receive sufficient information from GHP, half (8/16) wanted more information or a plan from GHP. Three of these eight PCPs had patients with negative results and requested more information on ES testing methodology. The remaining five PCPs with variants detected requested more communication and information regarding management of the patient and family. Of the other eight PCPs who did not receive sufficient information, five $\mathrm{PCPs}$ indicated that they never received the ES results and two did not specify the types of additional information they required. One PCP with a negative result stated that "I don't think that this [genetics information] should be put on the primary care provider."

Primary care physicians were asked about their expectations for their patient's follow-up care and responsibilities of the PCP, GHP, and the patient's family (Table 3). The majority of PCPs agreed with the statements that their patient will receive follow-up care from GHP (20/27, 74\%), GHP will update the patient and their family with new information about the ES result as it becomes available (19/27, 70\%), and GHP will update the PCP with new information about the ES result as it becomes available (15/27, 56\%). More PCPs agreed that it is the family's responsibility $(20 / 27,74 \%)$, rather than the PCP's responsibility (12/27, 44\%) to follow-up with GHP about new ES result information as it becomes available. Similar numbers of PCPs agreed that it is the family's responsibility $(17 / 27,63 \%)$ and the PCP's responsibility (16/27, 59\%) to follow-up with GHP about the patient's medical care.

\section{4 | DISCUSSION}

This study highlights areas where communication and collaboration between PCPs and GHP can be strengthened to improve continuity of care for genetics patients.

\begin{tabular}{|c|c|c|c|c|}
\hline \multirow[b]{2}{*}{ Communication expectations } & \multicolumn{4}{|c|}{ Type of exome sequencing (ES) result received } \\
\hline & $\begin{array}{l}\text { Pathogenic } \\
(n=10)\end{array}$ & VUS $(n=12)$ & $\begin{array}{l}\text { Negative } \\
(n=5)\end{array}$ & $\begin{array}{l}\text { All results } \\
(n=27)\end{array}$ \\
\hline $\begin{array}{l}\text { Patient will receive follow-up } \\
\text { care from GHP }\end{array}$ & $8 / 10(80 \%)$ & $10 / 12(83 \%)$ & $2 / 5(40 \%)$ & $20 / 27(74 \%)$ \\
\hline $\begin{array}{l}\text { GHP will update the pa- } \\
\text { tient with new ES result } \\
\text { information }\end{array}$ & $8 / 10(80 \%)$ & $9 / 12(75 \%)$ & $2 / 5(40 \%)$ & $19 / 27(70 \%)$ \\
\hline $\begin{array}{l}\text { GHP will update the PCP with } \\
\text { new ES result information }\end{array}$ & $6 / 10(60 \%)$ & $8 / 12(67 \%)$ & $1 / 5(20 \%)$ & $15 / 27(56 \%)$ \\
\hline $\begin{array}{l}\text { PCP received sufficient infor- } \\
\text { mation from GHP to properly } \\
\text { manage and counsel patient }\end{array}$ & $4 / 10(40 \%)$ & $6 / 12(50 \%)$ & $1 / 5(20 \%)$ & $11 / 27(41 \%)$ \\
\hline \multicolumn{5}{|c|}{ Responsibility to follow-up with GHP about new ES result information } \\
\hline Family's responsibility & $6 / 10(60 \%)$ & $10 / 12(83 \%)$ & $4 / 5(80 \%)$ & $20 / 27(74 \%)$ \\
\hline PCP's responsibility & $6 / 10(60 \%)$ & $5 / 12(42 \%)$ & $1 / 5(20 \%)$ & $12 / 27(44 \%)$ \\
\hline \multicolumn{5}{|c|}{ Responsibility to follow-up with GHP about patient's medical care } \\
\hline Family's responsibility & $7 / 10(70 \%)$ & $8 / 12(67 \%)$ & $2 / 5(40 \%)$ & $17 / 27(63 \%)$ \\
\hline PCP's responsibility & $6 / 10(60 \%)$ & $8 / 12(67 \%)$ & $2 / 5(40 \%)$ & $16 / 27(59 \%)$ \\
\hline
\end{tabular}

TABLE 3 Primary care physicians (PCPs) experiences with genetic healthcare providers (GHP)

Note: Data presented in this table (Percentages) indicate the number of participants in each ES result category that agreed with the assessment statement. " $n$ " in each column equals the number of PCPs with the PCP reported result type. 


\section{1 | Medical home}

The Genetics in Primary Care Institute, an American Academy of Pediatrics Health Initiative, identifies providing a medical home as one of seven key roles a PCP maintains for genetics patients (Genetics in Primary Care, 2018). As the medical home, the PCP provides important continuous, accessible, and comprehensive family-centered care coordination for genetics patients with complex healthcare management plans, including a responsibility of discussing results as they are returned from specialists (American Academy of Pediatrics Council on Children With Disabilities, 2005; Genetics in Primary Care, 2018; Medical Home Initiatives for Children With Special Needs Project Advisory Committee \& American Academy of Pediatrics, 2002). PCPs are also being encouraged to incorporate genetics roles into their regular care and management of pediatric patients, including communication with families, making appropriate referrals, and managing care (Scott, \& Trotter, 2013). This broadened role for PCPs is greatly needed as genetic medicine continues to grow and the GHP shortage continues. Support for this expanding role stems in part from the difficulty PCPs and patients face in gaining access to genetics clinics (Tarini, Zikmund-Fisher, Saal, Edmondson, \& Uhlmann, 2015). Additional barriers to GHP, including clinic locations, long wait times, GHP shortage, and insurance coverage of tests, may also be impacting PCPs' willingness to both refer patients to GHP and to take on additional genetics responsibilities in their clinics (Mikat-Stevens et al., 2015; Tarini et al., 2015; Tetreault et al., 2015).

Primary care physicians appear comfortable discussing the importance of ES results, with $69 \%$ of PCPs encouraging patients' parents to discuss ES results with family. PCPs' willingness to discuss results importance with patients' parents may be due to their understanding of the clinical utility of ES for their patients and the relevance of results that are reported (Nguyen \& Charlebois, 2015). Our results deepen understanding of PCPs' needs and indicate they look to GHP to communicate results and management directly with the family versus coordinating through the PCP as medical home. This suggests that involvement and collaboration of multiple providers, GHP and PCP, are needed to create an informed medical home to support and manage the patient.

\subsection{Genetic technology}

With rapid genetic testing technology development, literature suggests that PCPs anticipate genome sequencing (GS) reaching clinical utility in the near future (Vassy et al., 2015). Advancing technology will require PCPs to develop understanding of the types of genetic tests utilized by GHP and the potential types of results received. This will be challenging as PCPs still lack understanding of ES technology and results. While recent literature describes PCPs' and cardiologists' views of an inevitable clinical use of GS, the minority of PCPs in the negative and VUS groups in our study indicated that future genetic testing would occur (Vassy et al., 2015). This suggests that our participants may view ES as the last possible genetic test for patients either currently or while failing to consider future test developments, such as clinical use of GS. Additionally, our results suggest that PCPs believe if ES does not discover a diagnosis, no underlying genetic etiology for the patient's symptoms exists, as most PCPs with negative results indicated that there is not a genetic cause for their patient's symptoms. These results suggest that PCPs may not fully understand the ES technology, what the next steps for their patients' diagnostic analysis may be, and how new genetic tests could be utilized in the future.

Although we found that PCPs may not completely understand ES technology, the majority found ES beneficial to their patient's care and identified positive impacts of ES results, suggesting that they recognize the clinical utility of ES and see the benefits of ES for the majority of patients, regardless of result type. A recent study found that with the implementation of GS in clinical care, PCPs want to be able to explain GS results to patients and families, but were concerned about their lack of general genomic knowledge hindering their ability to manage this aspect of their patient's care (Christensen et al., 2016). A complementary study indicated PCPs recognize they have practical issues implementing ES in their clinic due to discomfort interpreting the many possible ES results (Nguyen \& Charlebois, 2015). As new genetic testing technology with increasingly complex results is implemented in patient care, PCPs will need to continue obtaining appropriate understanding of current clinical genetic testing, including clinical utility, test implications, and potential results.

\section{3 | Genetics education}

The increasing pressure to take on genetics related roles in primary care is concerning as the last genetics training for $33 \%$ of the PCPs in our study was $10+$ years ago, before ES was incorporated into clinical practice. The PCPs in our study also self-reported genetics knowledge of an average score of 23.2 in a range of 8-40. Recent efforts to enhance PCP genetics education and reforms of medical school genetics training may be taking effect, as we found that PCPs with more recent genetics trainings showed higher genetics knowledge scores and confidence scores (Dhar, Alford, Nelson, \& Potocki, 2012; Hagiwara, 2017; Plunkett-Rondeu, Hyland, \& Dasgupta, 2015; Vassy et al., 2014). However, since the minority of PCPs in our study were assuming responsibility for follow-up patient care and updating ES result information, there is still a need for ongoing genetics education in PCP clinical practice to address advances in genetics to assist with PCP confidence in follow-up care. This deficit is especially important in light of the increasing accessibility to genetic testing outside of clinical settings (Powell et al., 2012). In total, our results are consistent with previous studies and suggest that continuing genetics education for PCPs is key to improving genetic literacy, confidence performing genetics tasks, and continuity of care for pediatric genetics patients. On-going research assessing implementation of genetics education into primary care is necessary to optimize the process and ensure highest quality genetics-based care. 


\section{4 | Clinic communication}

Parents have previously reported receiving inconsistent or misleading information regarding their child's CMA report due to interactions with multiple healthcare providers, sometimes including differing interpretations of results (Reiff et al., 2012). Applying this finding to ES highlights that regular communication between GHP and PCPs is needed to facilitate consistent genetic test result explanations. Without this consistent understanding, ES results, especially VUS, can be problematic for providers and families processing new information about a possible diagnosis. Our data adds to this concern with $45 \%$ of PCPs with VUS results interpreting them as diagnostic for their patients, stating results explained their patients' symptoms and recommending additional familial genetic testing based on a VUS result. Furthermore, two PCPs incorrectly identified that their patients received VUS results even though they were provided with the result type clinical documentation from genetics and in the recruitment letter. This is notable as VUS results are considered uninformative for clinical decision making and should not guide patient care per American College of Medical Genetics and Genomics guidelines, though clinical judgement is used by individual GHPs when reviewing VUS results (Richards et al., 2015). ES complexity requires open conversation and partnership between PCPs and GHP to ensure consistent and accurate information is presented for appropriate patient care.

As $59 \%$ of the PCPs stated that they needed more information from GHP in order to properly manage and counsel their patients, additional importance should be placed on communication from GHP to PCPs. Often a single clinic letter serves as communication from GHP to both the patient and provider with education of genetic test results and medical recommendations (VandenBoom, Trepanier, \& Carmany, 2018). While guidelines exist for writing GHP letters to patients, guidelines for provider-provider letter communication are lacking (Baker, Eash, Schuette, \& Uhlmann, 2002). A recent study on this topic concluded that patients appreciated short, straightforward, and simple letters; this contrasts with healthcare professionals comments on the usefulness of lengthy and detailed letters (Roggenbuck et al., 2015). This aligns with requests from the eight PCPs who indicated they wanted more information, either on ES testing methodology or regarding management of the patient and family.

\section{5 | Study limitations}

In the absence of a validated instrument specific to our research questions, our study utilized one developed specifically for this research. It contained modified questions derived from validated instruments and was piloted with medical professionals of varying levels of expertise. The small sample and unequal numbers of result types from a low response rate make respondent bias possible, though variability is present in the received responses suggesting this pool provides sufficient diversity to identify a number of critical factors in how pediatric ES results are used. Additional impacting factors include PCP recruitment from a single institution and responding PCPs with possibly greater interest in genetics and therefore more genetics/ES knowledge than PCPs in general. Results could also be impacted by alternate clinical models and types of collaborative care. Due to small sample size, we were unable to evaluate confounding factors affecting PCPs with negative results, who trended toward lower knowledge and confidence. We chose to focus on PCPs in order to maintain clinical consistency and specialists' perspectives could differ. As this work was conducted with communication relying on the electronic medical record system, it was surprising that five PCPs indicated that they do not recall receiving their patient's result. This may have influenced the accuracy of this study, though we do not believe that the PCPs did not have access to the results as the electronic medical records provide the ability to obtain the result and documentation of result communication. Lastly, this study depended on participant recall which may differ from actual outcomes.

\section{6 | Practice implications}

This study's results emphasize areas to consider when GHP and PCPs communicate regarding shared patients. Our results indicate PCPs look to GHP to communicate results and management followup directly with the family versus coordinating through the PCP and suggest that PCPs may not fully understand the next steps or possibility of future test developments for their patient's diagnostic odyssey. As new genetic technology is implemented in patient care, PCPs will need to continue expanding their understanding of current genetic testing and GHP will need to help educate PCPs regarding advances. PCPs want more information from GHP and additional importance should be placed on ensuring clear, consistent, and regular communication of information and interpretations between GHP and PCPs. PCPs will continue to serve as the medical home and GHP need to ensure that sufficient information is provided to the PCPs for the long-term management of their patients.

\section{7 | Research recommendations}

While PCPs receive genetics education in medical school, continued research on implementing and assessing genetics education in primary care is necessary to identify the optimal way to convey updated information regarding genetics into PCP practice. Additionally, as PCPs are requesting additional information from GHP for patient management, research evaluating how to improve communication between GHP and PCP is needed. This will provide guidance to GHP for ways of improving communication with providers that will improve patient collaborative care. This research would have an impact on care and management of patients evaluated by GHP in their medical home, especially as testing technology grows in accessibility and complexity.

\section{5 | CONCLUSION}

Optimizing ES utility requires effective communication and collaboration between PCPs and GHP. We assessed PCPs' understanding 
and utilization of ES results in pediatric patient management. Challenges in utilization of ES results and ongoing development of genetic technologies require strong communication between PCPs and GHP in order to ensure continuity of care for pediatric genetics patients. Our results suggest that PCPs are open to this interaction and highlight opportunities to improve genetic literacy, collaboration with GHP, and continuity of care for pediatric genetics patients.

\section{AUTHOR CONTRIBUTIONS}

Sarah Mazzola, Bridget O'Connor and Dr. Beverly Yashar contributed to completing this manuscript according to the following ICMJE criteria:

- Substantial contributions to the conception or design of the work; or the acquisition, analysis, or interpretation of data for the work; and

- Drafting the work or revising it critically for important intellectual content; and

- Final approval of the version to be published; and

- Agreement to be accountable for all aspects of the work in ensuring that questions related to the accuracy or integrity of any part of the work are appropriately investigated and resolved.

\section{ACKNOWLEDGEMENTS}

National Society of Genetic Counselors Research Special Interest Group and Michigan Association of Genetic Counselors, Inc. provided funding for this study. The research presented in this manuscript was conducted while Sarah Mazzola was obtaining a Masters in Genetic Counseling from the University of Michigan.

\section{COMPLIANCE WITH ETHICAL STANDARDS}

\section{Conflict of interest}

Sarah Mazzola, Bridget O'Connor, and Dr. Beverly Yashar declare that they have no conflict of interest.

\section{Human studies and informed consent}

All procedures followed were in accordance with the ethical standards of the responsible committee on human experimentation (institutional and national) and with the Helsinki Declaration of 1975, as revised in 2005. Informed consent was obtained from all participants for being included in the study.

\section{Animal studies}

No animal studies were carried out by the authors for this article.

\section{REFERENCES}

American Academy of Pediatrics Council on Children with Disabilities. (2005). Care coordination in the medical home: Integrating health and related systems of care for children with special health care needs. Pediatrics, 116(5), 1238-1244.

Baker, D. L., Eash, T., Schuette, J. L., \& UhImann, W. R. (2002). Guidelines for writing letters to patients. Journal Genetic Counseling, 11(5), 399-418.

Battaglia, A., Doccini, V., Bernardini, L., Novelli, A., Loddo, S., Capalbo, A., ... Carey, J. (2013). Confirmation of chromosomal microarray as a first-tier clinical diagnostic test for individuals with developmental delay, intellectual disability, autism spectrum disorders and dysmorphic features. Official Journal of the European Paediatric Neurology Society, 17, 589-599.

Biesecker, L. G., \& Biesecker, B. B. (2014). An approach to pediatric exome and genome sequencing. Current Opinion in Pediatrics, 26, 639-645.

Christensen, K. D., Vassy, J. L., Jamal, L., Lehmann, L. S., Slashinski, M. J., Perry, D. L., ... McGuire, A. L. (2016). Are physicians prepared for whole genome sequencing? A Qualitative Analysis. Clinical Genetics, 89(2), 228-234.

Coulter, M. E., Miller, D. T., Harris, D. J., Hawley, P., Picker, J., Roberts, A. E., ... Irons, M. (2011). Chromosomal microarray testing influences medical management. Genetics in Medicine, 13(9), 770-776.

Dhar, S. U., Alford, R. L., Nelson, E. A., \& Potocki, L. (2012). Enhancing exposure to genetics and genomics through an innovative medical school curriculum. Genetics in Medicine, 14, 163-167.

Genetics in Primary Care. (2018). American Academy of Pediatrics. Retrieved from https://www.aap.org/en-us/advocacy-and-policy/ aap-health-initiatives/genetics-in-primary-care/Genetics-in-YourPractice/Pages/default.aspx

Hagiwara, N. (2017). Application of active learning modalities to achieve medical genetics competencies and their learning outcome assessments. Advances in Medical Education and Practices, 8, 817-829.

Harvey, E. K., Fogel, C. E., Peyrot, M., Christensen, K. D., Terry, S. F., \& Mclnerney, J. D. (2007). Providers' knowledge of genetics: A survey of 5915 individuals and families with genetic conditions. Genetics in Medicine, 9(5), 259-267.

Henderson, L. B., Applegate, C. D., Wohler, E., Sheridan, M. B., HooverFong, J., \& Batista, D. A. (2014). The impact of chromosomal microarray on clinical management: A retrospective analysis. Genetics in Medicine, 16(9), 657-664.

Iglesias, A., Anyane-Yeboa, K., Wynn, J., Wilson, A., Truitt, M., Guzman, E., ... Chung, W. (2014). The usefulness of whole-exome sequencing in routine clinical practice. Genetics in Medicine, 16(12), 922-931.

Jez, S., Martin, M., South, S., Vanzo, R., \& Rothwell, E. (2015). Variants of unknown significance on chromosomal microarray analysis: Parental perspectives. Journal of Community Genetics, 6, 343-349.

Lantin-Hermoso, M. R., Berger, S., Bhatt, A. B., Richerson, J. E., Morrow, R., Freed, M. D., ... Beekman, R. H.; Section of Cardiology, Cardiac Surgery. (2017). The care of children with congenital heart disease in their primary medical home. Pediatrics, 140(5), e20172607.

Lazaridis, K. N., Schahl, K. A., Cousin, M. A., Babovic-Vuksanovic, D., Riegert-Johnson, D. L., Gavrilova, R. H., ... Farrugia, G.; Individualized Medicine Clinic Members. (2016). Outcome of whole exome sequencing for diagnostic odyssey cases of an individualized medicine clinic: The Mayo Clinic experience. Mayo Clinic Proceedings, 91(3), 297-307.

Lee, H., Deignan, J. L., Dorrani, N., Strom, S. P., Kantarci, S., QuinteroRivera, F., ... Nelson, S. F. (2014). Clinical exome sequencing for genetic identification of rare mendelian disorders. The Journal of the American Medical Association, 312(18), 1880-1887.

Medical Home Initiatives for Children With Special Needs Project Advisory Committee. American Academy of Pediatrics. (2002). The medical home. Pediatrics, 110(1), 184-186.

Mikat-Stevens, N. A., Larson, I. A., \& Tarini, B. A. (2015). Primary-care providers' perceived barriers to integration of genetics services: A systematic review of the literature. Genetics in Medicine, 17(3), 169-176. 
Miller, D. T., Adam, M. P., Aradhya, S., Biesecker, L. G., Brothman, A. R., Carter, N. P., ... Ledbetter, D. H. (2010). Consensus statement: Chromosomal microarray is a first-tier clinical diagnostic test for individuals with developmental disabilities or congenital anomalies. The American Journal of Human Genetics, 86, 749-764.

Nguyen, M. T., \& Charlebois, K. (2015). The clinical utility of wholeexome sequencing in the context of rare diseases-the changing tides of medical practice. Clinical Genetics, 88, 313-319.

Pearl, P. L., Pettiford, J. M., Combs, S. E., Heffron, A., Healton, S., Hovaguimian, A., \& Macri, C. J. (2011). Assessment of genetics knowledge and skills in medical students: Insight for a clinical neurogenetics curriculum. Biochemistry and Molecular Biology Education, 39(3), 191-195.

Plunkett-Rondeu, J., Hyland, K., \& Dasgupta, S. (2015). Training future physicians in the era of genomic medicine: Trends in undergraduate medical genetics education. Genetics in Medicine, 17, 927-934.

Powell, K. P., Christianson, C. A., Cogswell, W. A., Dave, G., Verma, A., Eubanks, S., \& Henrich, V. C. (2012). Educational needs of primary care physicians regarding direct-to-consumer genetic testing. Journal of Genetic Counseling, 21(3), 469-478.

Reiff, M., Bernhardt, B. A., Mulchandani, S., Soucier, D., Cornell, D., Pveritz, R. E., \& Spinner, N. B. (2012). "What does it mean?": Uncertainties in understanding results of chromosomal microarray testing. Genetics in Medicine, 14(2), 250-258.

Reiff, M., Giarelli, E., Bernhardt, B. A., Easley, E., \& Spinner, N. B., Sankar, P. L., ... Mulchandani, S. (2015). Parents' perceptions of the usefulness of chromosomal microarray analysis for children with autism spectrum disorders. Journal of Autism and Developmental Disorders, 45, 3262-3275.

Richards, S., Aziz, N., Bale, S., Bick, D., Das, S., Gastier-Foster, J., ... Rehm, H. L.; ACMG Laboratory Quality Assurance Committee. (2015). Standards and guidelines for the interpretation of sequence variants: A joint consensus recommendation of the American College of Medical Genetics and Genomics and the Association for Molecular Pathology. Genetics in Medicine, 17(5), 405-424. https://doi. org/10.1038/gim.2015.30

Rinke, M. L., Mikat-Stevens, N., Saul, R., Driscoll, A., Healy, J., \& Tarini, B. A. (2013). Genetic services and attitudes in primary care pediatrics. American Journal of Medical Genetics, 164A, 449-455.

Roggenbuck, J., Temme, R., Pond, D., Baker, J., Jarvis, L. M., .. Mendelsohn, N. J. (2015). The long and short of genetic counseling summary letters: A case-control study. Journal of Genetic Counseling, 24, 645-653.

Saam, J., Gudgeon, J., Aston, E., \& Brothman, A. R. (2008). How physicians use array comparative genomic hybridization results to guide patient management in children with developmental delay. Genetics in Medicine, 10(3), 181-186.
Saldaña, J. (2013). The coding manual for qualitative researchers (2nd ed, pp. 84-91). Los Angeles: SAGE Publications.

Sawyer, S. L., Hartley, T., Dyment, D. A., Beaulieu, C. L., Schwartzentruber, J., Smith, A., ... Boycott, K. M. (2016). Utility of whole-exome sequencing for those near the end of the diagnostic odyssey: Time to address gaps in care. Clinical Genetics, 89, 275-284.

Scott, J., \& Trotter, T. (2013). Primary care and genetics and genomics. Pediatrics, 132, S231-S237.

Tarini, B. A., Zikmund-Fisher, B. J., Saal, H. M., Edmondson, L., \& Uhlmann, W. R. (2015). Primary care providers' initial evaluation of children with global developmental delay: A clinical vignette study. The Journal of Pediatrics, 167, 1404-1408.

Tetreault, M., Bareke, E., Nadaf, J., Alirezaie, N., \& Majewski, J. (2015). Whole-exome sequencing as a diagnostic tool: Current challenges and future opportunities. Expert Review Molecular Diagnostics, 15(6), 749-760.

VandenBoom, E., Trepanier, A. M., \& Carmany, E. P. (2018). Assessment of current genetic counselor practices in post-visit written communications to patients. Journal of Genetic Counseling, 27, 681-688.

Vassy, J. L., Christensen, K. D., Lautenbach, D. M., Raghavan, S., Robinson, J. O., Blumenthal-Barby, J., ... McGuire, A. L. (2015). 'Someday it will be the norm': Physician perspectives on the utility of genome sequencing for patient care in the MedSeq Project. Personalized Medicine, 12(1), 23-32.

Vassy, J. L., Lautenbach, D. M., McLaughlin, H. M., Won Kong, S., Christensen, K. D., Krier, J., ... Green, R. C. (2014). The MedSeq Project: A randomized trial of integrating whole genome sequencing into clinical medicine. Trials, 15(85), 1-12.

\section{SUPPORTING INFORMATION}

Additional supporting information may be found online in the Supporting Information section at the end of the article.

How to cite this article: Mazzola SE, O'Connor B, Yashar BM. Primary care physicians' understanding and utilization of pediatric exome sequencing results. J Genet Couns. 2019;28:1130-1138. https://doi.org/10.1002/jgc4.1163 